\title{
What Are the Key Points of Treatment for Cases of Mild Cognitive Impairment? Based on the Evaluation of Cognitive Function Tasks in the ADASJcog
}

\author{
Yoshihiko Yoshii $^{a}$ Akiko Takahashi ${ }^{b}$ Miyuki Ishizawa ${ }^{b}$ \\ ${ }^{a}$ Cognitive Impairment Medical Center, Tsukuba Memorial Hospital, Tsukuba, Japan; ${ }^{b}$ Division of Clinical \\ Psychology, Tsukuba Memorial Hospital, Tsukuba, Japan
}

\section{Keywords}

Mild cognitive impairment · Alzheimer's Disease

Assessment Scale Japanese edition · Hierarchy of cognitive impairment · Factors for improving dementia

\begin{abstract}
Background/Aims: The aims of this study were to identify the degree of atrophy of the hippocampus in image findings and which cognitive function items should be focused on when treating mild cognitive impairment. Methods: A total of 66 cases with mild cognitive impairment were included in the study over a 1.5-year observation period. MR images were used to assess hippocampal atrophy, and cognitive function was assessed by the ADASJcog test. Results: In the mild dementia group, there was a hierarchical difference in the 4 cognitive impairments in which each degree was significantly higher hierarchically. In the normally improved group, memory and act dysfunction was significantly improved, and in deteriorated cases, memory, orientation, and act dysfunction increased significantly. The normally improved group tended to have lighter hippocampal atrophy than the deteriorating group. Discussion: In early treatment of mild cognitive impairment, it is important to focus on which cognitive items to treat, but there are no reports that present them numerically. Because it is not clear, there may
\end{abstract}

be a risk that dementia may progress due to stunned treatment. It became clear that it was meaningful to show it, and it was the orientation and act function. Conclusion: It was suggested that hippocampal atrophy should be kept within the normal range and that the key treatment was mainly to improve memory and act dysfunction without reducing orientation function.

(C) 2021 The Author(s)

Published by S. Karger AG, Basel

\section{Introduction}

DSM5 dementia diagnostic criteria are defined as one or more of complex attention, executive function, learning and memory, language, perception movement, and social cognitive impairment [1], and cognitive impairment other than memory impairment is also important. Alzheimer's dementia (subsequently abbreviated as AD) pathologically begins from the inside of the temporal lobe such as the hippocampus and develops into the parietal and lateral temporal lobes. In response to the pathological changes, cognitive impairment also appears from memory to visuospatial cognition, calculation, writing, and language, and it is known as the severity classification of dementia in Cummings and Benson $[2,3]$. karger@karger.com www.karger.com/dee

Karger $\stackrel{\text { ' }}{5}$

GOPEN ACCESS
(C) 2021 The Author(s)

Published by S. Karger AG, Basel

This is an Open Access article licensed under the Creative Commons Attribution-NonCommercial-4.0 International License (CC BY-NC) (http://www.karger.com/Services/OpenAccessLicense), applicable to the online version of the article only. Usage and distribution for commercial purposes requires written permission.
Correspondence to:

Yoshihiko Yoshii, yoshii_y@tsukuba-kinen.or.jp 
In $\mathrm{AD}$, the hierarchy of cognitive impairment is indicated along with the deterioration of cognitive symptoms, but there are no reports that show a quantity of the relationship between the degree of impaired items of each cognitive function and the degree of atrophy of the hippocampus about the symptom improvement of dementia. In the diagnostic imaging of dementia, the morphological analysis evaluation of $\mathrm{AD}$ by MRI suggests that strong atrophy of the hippocampus suggests cognitive decline $[4,5]$.

We retested ADASJcog after an average of about 1.5 years for cases diagnosed with normal or mild dementia levels by the first ADASJcog (Alzheimer's Disease Assessment Scale Japanese edition) test [6] at the time of initial diagnosis. In this report, (1) the relationship with each task of cognitive impairment was analyzed in patients with mild dementia diagnosed with the value at the time of re-examination; in addition, (2) the factors of deterioration and improvement were analyzed by comparing the case which changed from normal to mild dementia and the case which improved normally from mild dementia at the time of re-examination. We identified cognitive function tasks to be treated to improve cognitive symptoms and also identified their values.

\section{Cases and Methods}

\section{Case}

The subject was a case complaining of memory loss, and the same patient was tested for ADASJcog at the time of initial diagnosis, and the ADASJcog was retested after an average of about 1.5 years, and the value was compared and analyzed. Nineteen cases in the normal group (average age 77 years, average observation period 18.5 months, 11 men and 8 women) and 47 cases in the mild dementia group (average age 77 years, average observation period 19 months, 17 men and 30 women) that diagnosed with ADASJcog retest values were analyzed.

\section{Diagnostic Imaging Method}

The MR instrument used was Philips Achieva1.5T, and the voxel-based specific regional analysis system for Alzheimer's disease (VSRAD) used VSRAD-advance2 software. Using VSRAD performed at the initial diagnosis, VSRAD-Z values of 2 or more and ratio values of 5 or more were evaluated as clear atrophy.

\section{Cognitive Function Evaluation Method}

The evaluation of cognitive impairment used the Japanese version of ADAScog (ADASJcog) created by Honma et al. [6]. The diagnosis of the severity of dementia was made from the overall point of ADASJcog. In other words, 36-70 points were advanced dementia, 23-35 points were moderate dementia, 10-22 points were mild dementia, and 9 points or less were normal [6].
On the other hand, the evaluation of each cognitive impairment classifies the 11 items of the ADASJcog test into 4 areas: memory, orientation, language, and act. In the 4 areas, the overall evaluation points for memory loss were 27 points in total: word reproduction function decrease (10 points), word recognition function decrease (12 points), and the regeneration of test instruction decrease ( 5 points). This time, the immediate memory function was also included in the memory function item for convenient examination.

The overall evaluation point of language function decline is 20 points in a total of the speech (language) ability decrease (5 points), auditory understanding decrease ( 5 points), word recall ( 5 points), and finger and article name function decrease ( 5 points). The overall evaluation point of the action function decrease was 15 points in a total of the verbal command function decrease (5 points), the constructional praxis function decrease ( 5 points), and the ideational praxis function decrease ( 5 points), and the disorientation function decrease was 8 points only of the orientation function decrease (8 points).

The measured values of cognitive decline in every 4 regions were evaluated by the ratio (cognitive decline rate) to every total point. Since the total points of cognitive function items classified into each 4 items were not the same, the evaluation method called the decline rate was used because it could not be evaluated by absolute values to compare and compare each other between each item. For example, memory function decrease rate $(\%)=$ memory dysfunction measurement value/total memory dysfunction value $(27$ points $) \times 100$.

For patients who complained of mild cognitive impairment, including memory impairment, we evaluated ADASJcog as normal for 9 points or less and 10-22 points as mild dementia levels. This study evaluates cognitive impairment from ADASJcog values.

\section{Analysis Method}

In the case diagnosed with mild dementia at the time of ADASJcog re-examination, (1) the degree of the disorder between the items of cognitive function classified into 4 areas was analyzed. In addition, (2) we compared the cases of improvement from mild dementia in the normal group and the cases of worsening from normal to mild dementia in the mild dementia group at the time of retesting.

And, it was numerically evaluated which item of the cognitive function classified into 4 areas was related to the improvement and deterioration of the symptom. The analysis was examined using Excel's $t$ test with equal variance and 2 -sided distribution. Some used Excel's VAR test as an analysis of variance.

\section{Results}

(1) Study on the degree of cognitive impairment and the degree of impairment between each cognitive function item in the normal group and the mild dementia group: Table 1 shows the degree of cognitive impairment in 4 areas in the normal and mild dementia groups.

In the memory function decline rate, it was $21.8 \%$ in the normal group and $34.7 \%$ in the mild dementia group, 
Table 1. Cognitive decline rates in normal and mild dementia groups

\begin{tabular}{|c|c|c|c|c|}
\hline \multirow[t]{2}{*}{ Degree of dementia } & \multicolumn{4}{|c|}{ Tasks of cognitive function } \\
\hline & $\begin{array}{l}\text { decrease rate of } \\
\text { language function, } \%\end{array}$ & $\begin{array}{l}\text { decrease rate of } \\
\text { memory function, } \%\end{array}$ & $\begin{array}{l}\text { decrease rate of } \\
\text { act function, } \%\end{array}$ & $\begin{array}{l}\text { decrease rate of } \\
\text { orientation function, } \%\end{array}$ \\
\hline Normal group (19 cases) & $0.3(1.1)$ & $21.8(5.9)^{\mathrm{a}}$ & $7.4(5.4)^{b}$ & $3.3(8.2)^{c}$ \\
\hline Mild dementia group (47 cases) & $2.4(6.6)$ & $34.7(12.2)^{a}$ & $20.6(11.7)^{\mathrm{b}}$ & $32.4(19.7)^{\mathrm{C}}$ \\
\hline
\end{tabular}

Values are mean (SD). The decrease rate of cognitive function of 3 items was significantly increased in mild dementia compared to the normal group. The display of ${ }^{\mathrm{a}-\mathrm{c}}$ shows the $t$ test result between the corresponding items. ${ }^{\mathrm{a}-\mathrm{c}} p<0.01$.

Table 2. Decrease rate of cognitive function in the normal group

\begin{tabular}{|c|c|c|c|c|}
\hline \multirow[t]{2}{*}{ Degree of dementia } & \multicolumn{4}{|c|}{ Tasks of cognitive function } \\
\hline & $\begin{array}{l}\text { decrease rate of } \\
\text { language function, } \%\end{array}$ & $\begin{array}{l}\text { decrease rate of } \\
\text { memory function, } \%\end{array}$ & $\begin{array}{l}\text { decrease rate of } \\
\text { act function, } \%\end{array}$ & $\begin{array}{l}\text { decrease rate of } \\
\text { orientation function, } \%\end{array}$ \\
\hline Normal group (19 cases) & $0.3(1.1)^{c, d}$ & $21.8(5.9)^{a-c}$ & $7.4(5.4)^{a, d}$ & $3.3(8.2)^{a, b}$ \\
\hline
\end{tabular}

Values are mean (SD). In the normal group of cognitive function, the memory function decrease rate was the highest, followed by the action function decrease rate and then the orientation function decrease rate. The $p$ value showed 0.001 in this group by the Excel's VAR test as an analysis of variance. The display of ${ }^{\mathrm{a}-\mathrm{d}}$ shows the $t$ test result between the corresponding items. ${ }^{\mathrm{a}-\mathrm{d}} p$ value $<0.01$.

Table 3. Decrease rate of cognitive function in the mild dementia group

\begin{tabular}{lllll}
\hline Degree of dementia & \multicolumn{2}{l}{ Tasks of cognitive function } & & \\
\cline { 2 - 5 } & $\begin{array}{l}\text { decrease rate of } \\
\text { language function, \% }\end{array}$ & $\begin{array}{l}\text { decrease rate of } \\
\text { memory function, \% }\end{array}$ & $\begin{array}{l}\text { decrease rate of } \\
\text { act function, \% }\end{array}$ & $\begin{array}{l}\text { decrease rate of } \\
\text { orientation function, \% }\end{array}$ \\
\hline Mild dementia group (47 cases) & $2.4(6.6)^{\mathrm{a}, \mathrm{d}, \mathrm{e}}$ & $34.7(12.2)^{\mathrm{a}, \mathrm{b}}$ & $20.6(11.7)^{\mathrm{b}-\mathrm{d}}$ & $32.4(19.7)^{\mathrm{c}, \mathrm{e}}$ \\
\hline
\end{tabular}

Values are mean (SD). In the mild dementia group of cognitive function, the memory function decrease rate and the orientation function decrease rate were both the highest, followed by the action function decrease rate and then the language function decrease rate. The $p$ value showed 0.034 in this group by the Excel's VAR test as an analysis of variance. The display of ${ }^{\text {a-e }}$ shows the $t$ test result between the corresponding items. ${ }^{\text {a-e }} p$ value $<0.01$.

and in the act function decrease rate, it was $7.4 \%$ in the normal group and $20.6 \%$ in the mild dementia group, and in the orientation function decline rate, it was 3.3\% in the normal group and $32.4 \%$ in the mild dementia group, and in the language function decline rate, it was $0.3 \%$ in the normal group and $2.4 \%$ in the mild dementia group. Thus, the rate of cognitive decline of 4 items increased significantly with worsening symptoms (Table 1).

On the other hand, concerning the degree of impairment between each item of cognitive function in the normal group and the mild dementia group, the normal group had a high rate of cognitive decline in the order of memory function, then action function, and disorienta- tion function (Table 2). In the mild dementia group, the decrease rate of the memory function and the disorientation function were both high, and there was no significant difference between the two, but the memory function decrease rate was significantly different between the active function decrease rate and the language function decrease rate (Table 3). In the analysis between each item of cognitive impairment, the hierarchy was that the memory function decline rate and the orientation function decline rate were significantly higher than other cognitive decline rates, the act function decrease rate was seen lower than that, and the language dysfunction rate was lower than that. 
Table 4. Comparison of initial and retested values in the 5 cases who exacerbated from normal to mild dementia

\begin{tabular}{|c|c|c|c|c|}
\hline ADASJcog value & $\begin{array}{l}\text { decrease rate of } \\
\text { language function, \% }\end{array}$ & $\begin{array}{l}\text { decrease rate of } \\
\text { memory function, } \%\end{array}$ & $\begin{array}{l}\text { decrease rate of } \\
\text { act function, } \%\end{array}$ & $\begin{array}{l}\text { decrease rate of } \\
\text { orientation function, \% }\end{array}$ \\
\hline Initial test value & $0.4(0.89)$ & $21.2(5.8)^{\mathrm{a}}$ & $9.3(3.6)^{b}$ & $7.5(11.2)^{c}$ \\
\hline Retested value & $0.6(1.34)$ & $29.9(6.9)^{a}$ & $18.7(5.6)^{b}$ & $25(17.7)^{c}$ \\
\hline
\end{tabular}

Values are mean (SD). In this group, the decrease rate in act function was significantly increased in the average of retest compared with the initial value. And, the memory function decrease rate and the orientation function decrease rate tended to increase though there was no significant difference in the average of retest compared with the initial value. It should be noted that even if the initial value is low, it may deteriorate. The display of ${ }^{\mathrm{a}-\mathrm{c}}$ shows the $t$ test result between the corresponding items. ${ }^{\mathrm{a}} p$ value $=0.065 .{ }^{\mathrm{b}} p$ value $<0.05$. ${ }^{\mathrm{c}} p$ value $=0.098$.

Table 5. Comparison of initial and retested values in the 9 cases who improved from mild dementia to normal

\begin{tabular}{|c|c|c|c|c|}
\hline \multirow[t]{2}{*}{ ADASJcog value } & \multicolumn{4}{|c|}{ Tasks of cognitive function } \\
\hline & $\begin{array}{l}\text { decrease rate of } \\
\text { language function, \% }\end{array}$ & $\begin{array}{l}\text { decrease rate of } \\
\text { memory function, \% }\end{array}$ & $\begin{array}{l}\text { decrease rate of } \\
\text { act function, } \%\end{array}$ & $\begin{array}{l}\text { decrease rate of } \\
\text { orientation function, } \%\end{array}$ \\
\hline Initial test value & $0.22(0.44)$ & $36.1(11.5)^{\mathrm{a}}$ & $19.3(11.8)^{b}$ & $9.7(12.2)^{c}$ \\
\hline Retested value & $0.11(0.33)$ & $24.1(3.3)^{\mathrm{a}}$ & $7.4(6.2)^{\mathrm{b}}$ & $5.6(11.0)^{c}$ \\
\hline
\end{tabular}

Values are mean (SD). In this group, the memory function decrease rate and the act function decrease rate were significantly reduced in the retest value compared with the first value. There was no significant difference in the orientation function decrease rate between the initial value and the retest value. The display of ${ }^{\mathrm{a}-\mathrm{c}}$ shows the $t$ test result between the corresponding items. ${ }^{\mathrm{a}} p$ value $<0.01$. ${ }^{\mathrm{b}} p$ value $<$ $0.05 .{ }^{c} p$ value $=0.46$

(2) Numerical examination of cognitive symptom improvement and aggravation factors in cases where normal to mild dementia deteriorated and cases that were normally improved from mild dementia at the time of reexamination: in 5 cases (average age 72 years, average observation period 24 months, 2 men and 3 women) who deteriorated from normal cognitive function to mild dementia, the decrease rate in act function was significantly different between the average value of $9.3 \%$ (standard deviation: SD 3.6\%) at the initial test and the average of $18.7 \%$ (SD 5.6\%) at the time of retest, and it was a significant increase at the time of re-examination $(p<0.05)$.

In the memory function decline rate, there was no significant difference between the average of $21.2 \%$ (SD $5.8 \%)$ at the initial test and the average of $29.9 \%$ (SD 6.9\%) at the time of retest, but it increased $(p=0.065)$. There was no significant difference $(p=0.098)$ between the average of $7.5 \%$ (SD 11.2\%) at the initial test and the average of $25 \%$ (SD 17.7\%) at the time of retest in the rate of decrease in orientation function, but the values at the time of retest increased (Table 4 ).

Similarly, in 9 cases (average age 79.8 years, average observation period 18 months, 4 men and 5 women) which improved normally from mild dementia, the memory decrease rate was significantly different between the average of $36.1 \%$ (SD $11.5 \%$ ) at the initial test and the average of $24.1 \%$ (SD 3.3\%) at the time of retest, and it was a significant decrease at the time of retest $(p<0.01)$. In the act function decrease rate, there was a significant difference between the average of $19.3 \%$ (SD 11.8\%) at the initial test and the average of $7.4 \%$ (SD 6.2\%) at the time of retest, and it was a significant decrease at the time of retest $(p<0.05)$.

In the orientation function decline rate, the average of 9.7\% (SD 12.2\%) at the initial test and the average of 5.6\% (SD 11.0\%) at the time of retest were both low, and there was no significant difference between the two $(p=0.46)$ (Table 5). For 9 normalized patients with mild dementia, as in other mild dementia patients, lifestyle improvement guidance such as exercise and/or acetylcholine-degrading enzyme inhibitors were used. Interestingly, in all cases of the aggravation group, memory, act, and orientation functions were worse than the initial value, and on the other hand, in all cases of the improvement group, those 3 functions were improved from the first value. 
Table 6. Comparison of VSRAD evaluation in the group which exacerbated from normal to mild dementia and in the group that was normally improved from mild dementia

\begin{tabular}{lll}
\hline VSRAD assessment & Degree of dementia & \\
\cline { 2 - 3 } & $\begin{array}{l}\text { mild dementia from normal } \\
\text { deteriorated group (5 cases) }\end{array}$ & $\begin{array}{l}\text { normal from mild dementia } \\
\text { improved group (9 cases) }\end{array}$ \\
\hline VSARD-Z value & $1.92(0.77)^{\mathrm{a}}$ & $\begin{array}{l}1.54(0.71)^{\mathrm{a}} \\
\text { VSARD-ratio value }\end{array}$ \\
$\begin{array}{ll}11.6(7.2)^{\mathrm{b}} & 5.5(3.97)^{\mathrm{b}}\end{array}$
\end{tabular}

Values are mean (SD). There was significant difference in VSRAD-ratio values in both groups; therefore, in the normally improved group, the hippocampal atrophy tended to be lighter than that of the deteriorated group. The display of ${ }^{\mathrm{a}, \mathrm{b}}$ shows the $t$ test result between the corresponding items. ${ }^{\mathrm{a}} p$ value $=0.27 .{ }^{\mathrm{b}} p$ value $<0.05$.
In the MRI at the initial diagnosis of both groups, the average VSRAD-Z value was 1.54 (SD 0.71), and the average ratio value was 5.5 (SD 3.97) in the group that improved normally from mild dementia. In the group that exacerbated from normal to mild dementia, the average $\mathrm{Z}$ value was 1.92 (SD 0.77), and the average ratio value was 11.6 (SD 7.2).

There was a significant difference in VSRAD-ratio values in both groups; therefore, in the normally improved group, the hippocampal atrophy tended to be lighter than that of the deteriorated group (Table 6). Unfortunately, we could not compare with the MR findings at the first time because MR images were not taken at the time of ADASJcog retest.

\section{Consideration}

(1) Study on the degree of cognitive impairment and the degree of impairment between each item of cognitive function in the normal group and the mild dementia group: it is known that hierarchy and locality are characteristics of higher brain function $[7,8]$. That is, it consists of a function which becomes the base function such as consideration, generality attention, and emotion in the bottom stage, a basic neurofunction such as movement, sight, and hearing in the next layer, an individual higher brain function such as language, an act, and calculation, and the top stage consists of functions that integrate various functions such as executive functions.

If lower functionality is strongly faulted, it is difficult to evaluate higher-level functions [7, 8]. In $\mathrm{AD}$, memory impairment is mainly in the early stage of the disease, but as the disease progresses, cognitive impairment is added to visuospatial dysfunction as well as calculations, writing, and language functions $[2,3]$.

$\mathrm{AD}$ also suggests the hierarchical existence of cognitive impairment, but in treating and rehabilitating dementia, we need to know which cognitive function tasks to focus on and how much to reduce the rate of cognitive decline, depending on the severity of dementia. However, since there is no literature that specifically clearly states them, we examined cognitive function tasks and the rate of decline.

In our results, there was a hierarchy that mild dementia levels started with a decrease in memory and orientation function, followed by a decrease in act function, and even a decrease in language function. Although our results were almost the same as a literature report, we would like to emphasize the importance of orientation function as well as memory function at mild dementia levels.

(2) Numerical examination of cognitive symptom improvement and aggravation factors in cases where normal to mild dementia deteriorated and cases that were normally improved from mild dementia at the time of re-examination: in our results in the group that improves normally from mild dementia, memory dysfunction and act function disorder were significantly improved at the time of retest; furthermore, it was characteristic that orientation dysfunction was very little at the first and second test.

On the other hand, in cases deteriorating from normal to mild dementia, in addition to the increase in the rate of memory dysfunction, the rate of orientation and act dysfunction also increased significantly. Therefore, it was suggested that as a treatment to improve mild dementia patients normally or not to worsen the degree of disease, it was not to reduce the orientation dysfunction but to improve memory and act dysfunction.

Specifically, it was suggested that the memory function decline rate should be referred to by $36 \%$, the orientation function decrease rate by $6 \%$, the act function decline rate by $19 \%$, and the language function decline rate by $0 \%$. As a nondrug treatment for dementia patients, it is said that reminiscence methods encourage brain activation [9].

In particular, rehabilitation for orientation and memory impairment has been reported to be useful in improving those dysfunctions $[10,11]$. In our results, the degree 
of disorientation is important, and treatments that do not promote memory impairment, act disorders, and disorientation are necessary. Although this study is within a limited observation period, it is significant that it suggests cognitive function items to be targeted when aiming to prevent dementia and improve from dementia level in the normal and mild dementia group.

Examination of hippocampal volume in the group that normally improved from dementia and the group that exacerbated from normal to mild dementia: in this study, there was no significant difference between the VSRAD$\mathrm{Z}$, but there was significant difference in VSRAD-ratio values in both groups; therefore, in the normally improved group, the hippocampal atrophy tended to be lighter than that of the deteriorated group. The hippocampal atrophy was within the normal range, and it tended to be considerably lighter than the group which deteriorated from normal to mild dementia.

\section{Conclusion}

Although the cognitive function evaluation in the ADASJcog test and the observation period were limited, in mild dementia, memory dysfunction and orientation dysfunction appeared mainly, followed by act dysfunction, and finally in the order of language dysfunction, there was a hierarchy in the appearance order and the strength of the disability. To normally improve mild dementia patients or not worsen the degree of disease, it was suggested that hippocampal atrophy should be kept within the normal range and that the treatment was mainly to improve memory dysfunction and act dysfunction without reducing orientation function. It was suggested that the memory function decrease rate should be referred to by $36 \%$, the orientation function decrease rate by $6 \%$, the action function decrease rate by $19 \%$, and the language function decline rate by $0 \%$.

\section{Statement of Ethics}

The subjects (or guardians) have given their written informed consent. This study has been approved by the Hospital Ethics Review Committee (H29-05-02, H29-05-03).

\section{Conflict of Interest Statement}

The authors have no conflicts of interest to declare. The corresponding author has completed the COI self-report to the Japanese Society of Neurosurgery. There is no COI to disclose regarding this study.

\section{Funding Sources}

None.

\section{Author Contributions}

The corresponding author collected, analyzed, and interpreted materials for the writing of this manuscript. The corresponding author will respond to the recommendations from the Editorial Office regarding this study. The other 2 co-authors (Akiko Takahashi and Miyuki Ishizawa) conducted the ADASJcog test as an expert in clinical psychology for all patients under the direction of the corresponding author.

\section{Data Availability Statement}

All data generated or analyzed during this study are included in this article. Further enquiries can be directed to the corresponding author.

\section{References}

1 Takahashi S, Oono Y. Ed. (Japanese). DSM5 mental illness classification and diagnosis manual. Japanese Society of Neuropsychiatry. Tokyo: Igakusyoin; 2014.

2 Dementia text book (Japanese), Japan Society for Dementia Research Edition. Tokyo, Cyugai Igakusya; 2012. p. 237-8.

3 Matsubara E, Syouji M, Abe K. Cummings, and Benson classifications. Nihon Rinsho (Japanese). Dementia. 2003;1:117-9.

4 Matsuda $\mathrm{H}$. The usefulness of MRI numerical analysis (VSRAD) in the diagnosis of Alzheimer's disease; Series "how to see dementia?" Cognition and Dementia. 2010;9(3):238-48.
5 Matsuda H. Diagnostic imaging of Alzheimer's disease; "Diagnostic treatment for Alzheimer's disease". JPN J Geriatr. 2012(49):425-30.

6 Honma A, Fukuzawa K, Tsukada Y, Ishii T, Hasegawa K, Mohs RC. Development of a Japanese version of Alzheimer's Disease Assessment Scale (ADAS). Nihon Ronen Igakukai Zasshi. 1992;3:647-55.

7 Suzuki K. The simple mental status examination. JPN J Neuropsychol. 2016;32:224-8.

8 Mori E, Mitani Y, Yamadori A. Usefulness of a Japanese version on the mini-mental state test in neurological patients. JPN J Neuropsychol. 1985;1:82-90.
9 Yamaguchi H. Brain activation rehabilitation of dementia. Proceeding of the Annual Meeting of the Japanese Research Group on Senile Dementia (Japanese). 2011;18:1339.

10 Kashima H, Yoshimasu K, Kato M Cognitive rehabilitation for mnestic and attentional disorders. JPN J Neuropsychol. 1997; 13:184-9.

11 Watamori T, Honda R. Rehabilitation of memory impairment: specific methods-rehabilitation medicine (Japanese). JPN J Rehabil Med. 2005;42(5):313-9. 